\title{
Pour un portail de veille informationnelle
}

\author{
Christian Bélanger ${ }^{1}$ \\ Université du Québec à Chicoutimi
}

1 e positionnement de la région du SaguenayLac-Saint-Jean et de l'ensemble de ses collectivités au sein de l'économie du savoir demande que cette dernière se dote d'outils permettant l'appropriation des technologies de l'information et de la communication ${ }^{2}$. Ce faisant, l'accès aux nouvelles connaissances, au développement de savoirs et à une bonification des savoir-faire existants ${ }^{3}$ en serait facilité. Il faut donc qu'elle procède à la mise en place d'un processus d'appropriation permettant à la fois d'utiliser les structures de retransmission des signaux porteurs d'information à distance ainsi qu'une plate-forme informatique, accessible à tout type d'utilisateur ${ }^{4}$ permettant la diffusion de données textuelles, visuelles et sonores.

La mise en place d'un portail de veille informationnelle constitue un outil d'appropriation des technologies de l'information et de la communication qui non seulement faciliterait l'échange des connaissances et des savoirs, mais qui contribuerait également au renforcement des initiatives déjà amorcées sur le territoire.

\section{Une élaboration basée sur les enseignements des processus existants}

Certaines collectivités québécoises ont procédé à la mise sur pied de différents processus d'appropriation des technologies de l'information et de la communication ${ }^{5}$ : tandis que des collectivités ont procédé à la mise en place de sites Internet municipaux, certaines collectivités ont, pour leur part, procédé à d'autres types d'expériences d'appropriation qui ont pris la forme de portails. L'analyse comparative de ces deux types d'expériences permet d'en tirer de précieux enseignements dont le projet de portail de veille informationnelle peut tirer avantage, notamment au plan des différents concepts inhérents à ce type de projet de même qu'au plan de la mise sur pied de ces derniers.

\section{Les organisations sollicitées pour ce projet et leur rôle}

Les organisations participant à ce type de processus peuvent être divisées ${ }^{6}$ en trois catégories, soit les maîtres d'œuvre (qui assument la direction du projet), les acteurs (des organisations participant au projet à différents niveaux ainsi qu'à sa direction) et les partenaires (qui assument plutôt un rôle n'impliquant pas la participation à un processus décisionnel, par exemple en fournissant toutes sortes de contenus). Ces organisations sont également de différents types, soit des représentants démocratiques (des instances généralement élues), des gestionnaires de service (la gestion de services gouvernementaux) ainsi que des acteurs stratégiques (procurant un soutien territorial aux acteurs économiques), tactiques (promotion des initiatives privées) ou exogènes (acteurs généralement extérieurs à la collectivité).

Le niveau de responsabilisation et le niveau de participation des organisations au processus de mise en place de type portail diffèrent de celui des sites Internet municipaux: leur structure de fonctionnement favorise une plus grande participation des différentes organisations. Ils peuvent aussi être pris en charge par une plus grande variété de maîtres d'œuvre. Le niveau de participation des différents types d'acteurs s'avère plus élevé de même que le niveau de participation et la provenance des partenaires. Il permet également aux organisations d'assumer des tâches ou des fonctions plus variées qu'au sein des sites Internet municipaux. 
Nous pouvons déjà déterminer qu'en ce qui a trait au leadership du nouvel outil d'appropriation des TIC, ce dernier n'est pas lié à un type d'organisation en particulier. Cependant, il faut noter que le maître d'œuvre d'un tel projet doit posséder certaines qualités inhérentes au rôle qu'il doit assumer : les capacités de mobilisation des acteurs de procéder à une alimentation en contenu, de mettre en place des infrastructures de fonctionnement, de procéder à une offre de services, d'assurer une cohésion des organisations participantes et d'obtenir des ressources nécessaires au fonctionnement du projet sont des critères déterminants ${ }^{7}$.

Le choix des acteurs ainsi que des partenaires participant à la mise en place du portail de veille informationnelle est tributaire des finalités organisationnelles visées par le portail de veille informationnelle. Puisque ce dernier se veut être un outil d'appropriation des technologies de l'information et de la communication permettant l'échange des connaissances, des savoirs et le renforcement des initiatives déjà amorcées, il apparaît clairement que ce dernier doit travailler au plan des quatre finalités organisationnelles permettant d'enrichir le soutien au développement ${ }^{8}$, soit le marketing territorial (communication d'information variée au sujet de la collectivité), la réponse aux besoins de base de la population (mise en place de services bénéficiant à la collectivité), la veille collective (l'accès à une information de pointe sur différents domaines) ainsi que la combinaison appropriée des conditions (conditions matérielles ou immatérielles permettant le soutien du développement de la collectivité). Donc, il s'avère impossible de déterminer le choix des organisations pouvant agir à titre d'acteurs ou de partenaires du portail de veille informationnelle. Le déterminer équivaudrait à limiter le nombre d'organisations pouvant participer au processus, bloquant, ou du moins retardant, l'accès aux connaissances ou au développement du savoir dans un domaine spécifique ou au niveau d'une des finalités organisationnelles. Un appel ouvert à l'ensemble des organisations existantes sur le territoire doit donc être effectué, peu importe à quel type de catégorie d'organisations ils appartiennent.

\section{Les stratégies à adopter}

Les projets de type portail appuient l'atteinte des finalités organisationnelles par des stratégies et des res- sources appropriées ${ }^{9}$. Le choix des stratégies à adopter permettra de déterminer quelles seront les ressources à employer pour les atteindre. Nous pouvons déjà avoir une idée exacte de certaines des stratégies à adopter en vue d'appuyer les finalités organisationnelles du portail de veille informationnelle en tenant compte des résultats d'une analyse réalisée en mai 2004 portant sur les sites Internet ${ }^{10}$ existant sur le territoire du Saguenay-Lac-Saint-Jean.

\section{Faiblesses des sites Internet régionaux étudiés}

Les sites Internet régionaux ne bénéficient pas de la participation d'une très grande variété d'organisations. Il est intéressant de noter que ces mêmes sites Internet se spécialisent au sein d'une ou deux finalités organisationnelles : cette spécialisation limite les retombées pouvant être tirées de l'utilisation des technologies de l'information et de la communication (TIC). Aussi, il ne semble exister aucune coordination permettant de tirer le maximum des TIC. Par ailleurs, on note la présence de différentes faiblesses telles que le manque de liens entre les sites Internet régionaux, une alimentation en contenu déficiente, un manque de ressources qualifiées pour l'entretien de ces sites et un support public limité.

\section{Le portail doit travailler au plan des quatre finalités organisationnelles permettant d'enrichir le soutien au développement.}

Il faut donc, d'une part, procéder à la mise en place des stratégies permettant de contourner l'ensemble des difficultés rencontrées par les sites Internet régionaux étudiés qui viendront, d'autre part, appuyer le portail de veille informationnelle dans l'atteinte des quatre finalités organisationnelles. La stratégie suivante permet de contourner ces difficultés et d'appuyer l'atteinte des quatre finalités organisationnelles.

\section{La veille sur demande}

Cette stratégie permet la coordination de la diffusion des savoirs produits par les organisations participantes : l'envoi des informations sous forme de courriels à un ensemble de personnes abonnées à différentes listes d'envoi thématiques mises en place à partir du portail de veille informationnelle. 
L’exemple suivant illustre la façon de procéder : supposons qu'une ville ou un village ait produit un rapport décrivant le potentiel de développement des PME sur son territoire. Un responsable dûment nommé fait part de la disponibilité de l'information sur la liste municipalités-1@vdemande.com. Ainsi, cette information est expédiée à l'ensemble des gens abonnées à cette liste. La possibilité de créer des listes thématiques peut être très grande si l'on en juge par la présence de nombreuses organisations présentes dans la région qui œuvrent dans des champs d'action allant du développement local au tourisme, des municipalités aux organisations communautaires, des chaires de recherches aux commissions scolaires.

Le portail de veille informationnelle permettra donc d'offrir aux personnes ou aux organisations le désirant la possibilité de s'abonner à une ou des listes thématiques, suivant leur champ d'intérêt, afin de disposer de l'information requise sans avoir à amorcer un processus de recherche les conduisant sur plusieurs portails puisque toute l'information voulue sera disponible en un point central, celui du portail de veille informationnelle.

\section{Avantages de cette stratégie}

Cette stratégie de veille sur demande permet l'atteinte de l'ensemble des finalités organisationnelles: elle crée une collaboration entre les organisations de la région du Saguenay-Lac-Saint-Jean qui, malgré la diversité de leur activité, peuvent travailler sur un projet commun, celui de l'appropriation des savoirs et des connaissances. Elle permet également le partage des savoirs et des connaissances, elle vient en appui du développement économique et social régional en rendant accessibles les savoirs et connaissances non seulement aux individus, mais également aux organisations. En outre, elle crée un portail régional collectif où les différentes thématiques traitées par les organisations régionales se retrouvent et peuvent être accessibles, permettant du même coup de faire la promotion du territoire et de ses éléments.

Tout en permettant l'atteinte des différentes finalités organisationnelles, la stratégie de veille sur demande permet aussi de contourner différents obstacles qui sont connus chez certains sites Internet régionaux, à savoir la participation des différentes organisations du territoire, une trop grande spécialisation du portail, une coordination des initiatives (la façon dont les personnes et les organisations ont accès aux contenus informationnels), le manque de liens entre les sites Internet régionaux (la participation au portail de veille informationnelle donne l'occasion aux différentes organisations d'échanger à propos de leur initiative TIC respective) ainsi que l'alimentation en contenu. Puisque le portail de veille informationnelle sert de plate-forme d'abonnement aux listes d'envois par courriel, l'alimentation en contenu n'est plus un problème du portail a priori, une section d'archivage de tous les contenus pouvant être mise en place pour permettre l'accès aux contenus déjà postés si jamais un utilisateur s'abonne à la suite d'un envoi important.

De plus, le manque de ressources qualifiées pour l'entretien du site devient un moindre problème étant donné que le portail est utilisé à titre de plate-forme où les personnes et les organisations peuvent s'abonner à des listes d'envoi thématiques (la majorité des contenus informationnels transitant donc par courriel). Quant au support fourni par les pouvoirs publics, la réponse que les organisations de ce secteur fourniront permettra de jauger le niveau d'implication et d'intérêt de ces dernières vis-à-vis du nouvel outil d'appropriation des TIC que la collectivité régionale sera appelée à mettre en place.

\section{Cette stratégie de veille sur demande permet l'atteinte de l'ensemble des finalités organisationnelles.}

\section{D’autres stratégies appuyant la veille sur demande}

Pour permettre l'optimisation des retombées obtenues par la stratégie de veille sur demande, il importe d'adopter trois autres stratégies complémentaires permettant à la fois d'accéder aux différents contenus informationnels et de les consulter.

\section{L'accès au réseau Internet}

Si la stratégie de veille sur demande permet la diffusion de différents contenus informationnels, il va sans dire qu'il faut permettre à la population d'avoir accès à ces contenus afin d'éviter la création d'une « fracture numérique ${ }^{11}$ régionale. Il est possible de rendre accessibles les contenus informationnels en établis- 
sant des ententes avec les Centres d'accès communautaires à Internet puisque plusieurs collectivités de la région possèdent un CACI sur leur territoire, ce qui permettrait une porte d'entrée pour accéder aux contenus du portail de veille informationnelle.

Il est également possible de rendre les contenus accessibles en procédant au branchement des «nonbranchés », qu'ils soient des personnes ou des organisations. Une stratégie de branchement, à un prix avantageux, permettrait d'inciter ces derniers à bénéficier des contenus informationnels.

\section{I l est possible que, dans certains milieux, les clientèles soient moins habilitées à se servir de l'I nternet et du courriel.}

\section{Programme d'achat d'ordinateurs}

La mise en place d'un programme d'achat d'ordinateurs doit être développée afin de rendre possible l'accès, à un coût acceptable, de ressources informatiques pour les individus et les organisations ne disposant pas de ce matériel. De plus, il faudra tenir compte d'une autre donnée, soit celle de l'entretien de ces derniers.

Pour ces deux éléments, des partenariats avec des fournisseurs peuvent être développés étant donné la présence élevée des fournisseurs potentiels au sein de la région. Il sera du devoir des organisations participant à la direction du portail de veille informationnelle de voir à la mise en place d'un tel programme afin de ne pas créer une "fracture numérique », tel que mentionnée au point précédent.

\section{Formation de la population et des organisations}

Il est possible que, dans certains milieux, les clientèles soient moins habilitées à se servir de l'Internet et du courriel. Une formation de groupe peut être offerte aux différentes personnes qui en exprimeraient le besoin. Elle pourrait même être offerte de façon personnalisée et se déplacer au domicile ou dans l'organisation des utilisateurs ${ }^{12}$ : un moniteur ou une monitrice, disposant d'un ordinateur portable et de l'équipement approprié, se déplacerait au domicile ou dans l'organisation des gens qui en font la demande.

\section{Les étapes à franchir pour la mise en place du portail}

Le choix du maître d'œuvre est le point de départ de la mise en place du portail de veille informationnelle. Nous avons déjà traité des qualités dont ce maître d'œuvre devra disposer. L'un des premiers mandats auxquels il devra travailler est celui du financement nécessaire à la mise en place du projet de même qu'à sa pérennité.

Par la suite, il sera nécessaire de voir à la participation d'un premier acteur dont la participation sera cruciale: un expert provenant du domaine de l'informatique, permettant ainsi d'obtenir l'accès au réseau Internet, à des listes d'envoi de courriels ainsi qu'à un hébergement du portail. À partir du moment où cet acteur sera connu, il sera possible de voir quels sont les autres besoins de partenariat pour la mise en place du projet, advenant le cas qu'un seul acteur ne puisse fournir les ressources requises.

Une fois l'armature numérique du projet assurée, le maître d'œuvre devra procéder à l'établissement d'un diagnostic portant sur les aspects de la population et des organisations régionales. Ce portait permettra :

- de connaître le niveau d'intérêt de la population à bénéficier d'un tel service, en plus de connaître les différents facteurs de résistance à la mise en place du projet;

- de connaître le nombre d'organisations présentes sur le territoire et leur secteur d'activité de même que leur intérêt à participer au portail de veille informationnelle, à en bénéficier, la procédure de fonctionnement désirée et les différents facteurs de résistance à leur participation au projet.

Ces deux aspects permettront de cibler les points précis sur lesquels le maître d'œuvre ainsi que les différents acteurs et partenaires du projet auront à travailler afin d'en assurer la réussite.

Une fois que les points de travail seront établis, le maître d'œuvre du projet devra se mettre en devoir de rallier les différentes organisations présentes sur le territoire autour du portail de veille informationnelle. Pour ce faire, il devra les rencontrer afin de leur présenter le portail de veille informationnelle, ses buts et objectifs, la procédure de fonctionnement et ce que 
signifie l'implication des organisations en termes d'engagement.

La mise en place d'un bureau de direction du projet sera nécessaire à la bonne marche du projet : bien que le maître d'œuvre possède différentes qualités inhérentes au lancement du portail de veille informationnelle, un bureau de direction permettra que des organisations régionales participent aux prises de décisions touchant le portail de veille informationnelle, aux diverses stratégies de travail que le portail devra adopter de même qu'aux différents aspects du projet (financement, plate-forme Web, etc.). De plus, ce bureau de direction permettra l'établissement d'une structure participative pour les organisations, ne les confinant pas au rôle de fournisseur de contenu, mais leur permettant de s'approprier le projet, assurant ainsi une viabilité du projet si l'on en croit les expériences des différents projets de portail ayant eu lieu sur le territoire québécois.

La composition de ce bureau de direction inclut tout d'abord le maître d'œuvre du portail de veille informationnelle. Ce dernier, tout en assumant un rôle de direction, pourra coordonner le projet. L'inclusion de l'acteur fournissant l'infrastructure numérique permettra aux organisations participant à la direction du projet de connaître l'état du réseau et de reconnaître l'importance de la participation de cet acteur au projet. Pour ce qui est du reste de la composition du bureau de direction du projet, il s'agit pour le maître d'œuvre d'effectuer une démarche auprès de l'ensemble des acteurs participants tout en gardant à l'esprit l'importance de la représentativité de ces derniers. Les finalités organisationnelles du projet permettront de viser les organisations participantes; ainsi, on pourra s'assurer de la présence d'au moins un acteur de chacune des sphères de finalités organisationnelles.

Le portail de veille informationnelle permettra à la région du SaguenayLac-Saint-J ean de posséder un outil collectif de maîtrise des technologies de l'information et de la communication.

À la suite de la composition du bureau de direction du projet, les organisations y participant pourront travailler à l'établissement des procédures de gestion du projet de même qu'aux stratégies à établir et à poursuivre pour le bon fonctionnement de ce dernier.

\section{Conclusion}

Le portail de veille informationnelle permettra à la région du Saguenay-Lac-Saint-Jean de posséder un outil collectif de maîtrise des technologies de l'information et de la communication. L'utilisation de ces technologies en tant que canal de retransmission des différents savoirs et connaissances produits par les organisations régionales, au moyen de la stratégie de veille sur demande, permettra d'impliquer les organisations participantes au sein du processus d'appropriation des TIC. Sans prétendre que le portail de veille informationnelle sera en mesure d'être LA solution pour ce qui est de l'appropriation des technologies de l'information et de la communication pour l'ensemble de la collectivité régionale, on peut toutefois affirmer que ce projet permettra une avancée très intéressante dans cette direction.

\section{Notes et références}

1 L'auteur est titulaire d'une maîtrise en études et interventions régionales de l'Université du Québec à Chicoutimi dont le sujet de mémoire était «Les apports des expériences d'appropriation des TIC par les collectivités québécoises ». Il entreprendra, à l'automne 2005, un doctorat en développement régional à la même institution.

2 Le terme de «technologies de l'information et de la communication » fait référence à la définition de «nouvelles technologies de l'information », plusieurs autres termes pouvant également être logés sous le même chapeau (source : Grand dictionnaire technologique de l'Office québécois de la langue française.

(http://www.granddictionnaire.com)

3 En 1994, le Conseil de la science et des technologies du Québec faisait état du constat au niveau québécois dans " Miser sur le savoir », Rapport de conjoncture 1994, Les nouvelles technologies de l'information, Conseil de la science et de la technologie, Gouvernement du Québec, 1994, p. 11-12.

4 Source: Grand dictionnaire terminologique de l'Office québécois de la langue française.

5 Les collectivités ayant mis sur pied des portails sont celles de l'arrondissement Hochelaga-Maisonneuve (Arrondissement.com), de la ville de Bromont (Bromont.com), du territoire de la MRC de Bécancour (Bécancour.net), de la région de la Mauricie (Réseau à large bande de la Mauri- 
cie) ainsi que de la région du Bas-Saint-Laurent (Technopole maritime du Québec). Les collectivités du deuxième type sont celles des villes d'Amos, Asbestos, BaieComeau, Gatineau, Joliette, Rimouski, Laval, Montréal, Québec, Rivière-du-Loup, Rouyn-Noranda, Sept-Îles, Shawinigan et Sherbrooke (source : Proulx, M.-U., L. Darhouani, J. Gauthier, A. Doubi, C. Bélanger et M.-A. Vachon (2003), Appropriation des TIC par les acteurs territoriaux du développement, rapport final présenté au CEFRIO, novembre, 134 p.).

Proulx, M.-U. et al. (2003), op. cit., p. 56-58.

$7 \quad$ Idem, p. 67.

8

9 Idem.
10 Sites de 23 municipalités du Saguenay-Lac-Saint-Jean, de Vision Saguenay 2025, les sites Internet des CLD et des SADC de la région 02, de l'ATR, de Tourisme Lac-SaintJean, Pilote02, la Toile du Royaume et la Toile du Saguenay (présentation «Idéal-type pour une meilleure appropriation des TIC au Saguenay-Lac-Saint-Jean », par M.-U. Proulx, Ph.D, dans le cadre d'un colloque sur les TIC, au Holiday Inn de Jonquière, le 2 juin 2004).

11 Ce problème de fracture numérique fait référence à la création de deux catégories de citoyens : ceux qui ont accès et ceux qui n’ont pas accès. Il est évoqué sur le portail de la collectivité de Joinville-Le-Pont.

(http://www.joinville-le-pont.fr.)

12 On se base sur une expérience du groupe communautaire ÉCOF de Trois-Rivières (en collaboration avec EmploiQuébec), qui avait cours en 2004. 